\title{
Management Colorectal Gastrointestinal Stromal Tumors (Gists) in Surabaya
}

\author{
Yuda Handaya1, Sutamto Wibowo'2, Iwan Kristian² \\ ${ }^{1}$ Departments of Gastrointestinal Surgery, Sardjito Hospital, Gadjah Mada Medical University, \\ Yogyakarta, Indonesia \\ ${ }^{2}$ Departments of Digestive Surgery, Sutomo Hospital University of Airlangga, Surabaya, Indonesia \\ Email: yudahandaya@yahoo.com
}

Received 3 October 2013; accepted 10 April 2016; published 13 April 2016

Copyright (C) 2016 by authors and Scientific Research Publishing Inc.

This work is licensed under the Creative Commons Attribution International License (CC BY). http://creativecommons.org/licenses/by/4.0/

(c) (i) Open Access

\begin{abstract}
Introduction: Colorectal gastrointestinal stromal tumors (GISTs) mesenchymal tumor is very uncommon. GISTs effect mostly on the stomach and small intestine and rarely occur in the colon, rectum and esophagus, that originating from precursors of the interstitial cells that originate of Cajal. The symptoms of gastrointestinal stromal tumor depend on the site and size of the tumor, and may include abdominal pain, gastrointestinal bleeding or signs of obstruction; small tumors may, however, be asymptomatic. Some of the patients with gastrointestinal stromal tumor have bloody stools, obstruction and abdominal pain as the commonest manifestation. Immunocytochemical staining for CD117 is helpful in confirming the diagnosis. Case presentation: We report 3 new cases of GISTs: two occurred at the rectal and the other at descending Colon. Two cases are over 50 years of age and, and all cases the chief complain of bowel obstruction, abdominal pain in two cases, and one case with anemia and urine retention. All the patients were operated and were permormed pathology examinatiom. All case ware positive result for immunocytochemical staining CD117. All cases we had presented had size more than $5 \mathbf{c m}$ are considered as unfavorable prognostic factors to Fletcher criteria, all patients scheduled for chemotherapy with Glivec but just one patient continued to used Glivec. Post surgery follows up one patient post milles with urinary incontinence complaints found and that patients are trained to CIC (intermittent catheterization). Conclusion: Colorectal gastrointestinal stromal tumors are very rare and can present as mass abdomen. Resection and chemotherapy are the treatment of choice.
\end{abstract}

\section{Keywords}

Colorectal, Gastrointestinal Stromal Tumors (GISTs), CD117 


\section{Introduction}

Gastrointestinal stromal tumors (GISTs) are the most common mesenchymal tumors of the gastrointestinal tract. Colonic and rectal gastrointestinal stromal tumors (GISTs) are very uncommon. Gastrointestinal stromal tumors (GISTs) are a heterogeneous group of mesenchymal tumors that arise from the gastrointestinal tract, and have been the cause of much confusion as to their origin, classification, biological behavior and prognosis. They have been called by many names such as STUMP (smooth muscle tumors of uncertain malignant potential), GANT (gastrointestinal autonomic nerve tumors), and GIPACT (gastrointestinal pacemaker cell tumors) in the past. However, advances in immunohistochemistry and electron microscopy have shown that these tumors originate from interstitial cells of Cajal (ICC) or stem cells differentiating towards the ICC. Seventy percent to $80 \%$ of GISTs are benign, and such tumors are often found incidentally at surgery and excised in the same session. Approximately $20 \%$ to $30 \%$ of GISTs are malignant, and these neoplasms are identified based on their mitotic index, tumor necrosis index, and Ki-67 index (which reflects expression of the 395 kDa nuclear antigen MIB-1) [1]-[5].

Histologically GISTs vary from spindle cell tumors to epithelioid and pleomorphic tumors. Most GISTs (95\%) express Kit (CD117), CD34 (70\%), and heavy caldesmon (80\%), whereas 25\% are positive for smooth muscle actin and less than $5 \%$ for desmin. GISTs differ histologically, immunohistochemically and genetically from leiomyomas, leiomyosarcoma and schwannomas. Fine needle aspiration can be used to diagnose GISTs as spindle cell and epithelioid types, but cytomorphology alone cannot be used to assess malignant potential. Immunocytochemical staining for CD117 is helpful in confirming the diagnosis. Care must be taken to differentiate epithelioid-type GISTs from adenocarcinoma. Surgery is the treatment of choice for resectable tumors. GISTs bear good prognosis after margin negative surgery. Tumor size and mitotic activity are best predictive prognostic features; small intestinal tumors behave more aggressively than gastric tumors with similar parameters [1] [2] [6].

\section{Case Report}

\subsection{Case Report 1}

A 70 year-old woman was admitted to hospital because of no defecation for 9 days and lower abdominal pain. Physical examination found abdominal distension and increasing peristaltic. Rectal examination find rectal polyp with diameter $8 \mathrm{~cm}$ the superior border not clear, colonoscopy cannot enter because of polyp mass.

Plain abdominal X-Ray (Figure 1) fined dilatation of intestine that mind shows an obstruction and we decided to performed urgent left transversocolostomy. And than we performed CT Scan are shown in Figure 2 looked in traluminar rectal tumor obstructed the rectum.

1 week after this diagnostic we performed laparotomy exploration is shown in Figure 3 find polyp mass that invasive on the rectal and sigmoid wall, decides to performed low anterior resection. Pathology result is myxoidliposarcoma without muscle and lymphoid gland infiltration. Immunocytochemical staining for CD117 is positive. Our programed is chemotherapy wit Glivec but Patient can find the drug because of no inventory. 1-year follow up patient have no defecation problem.

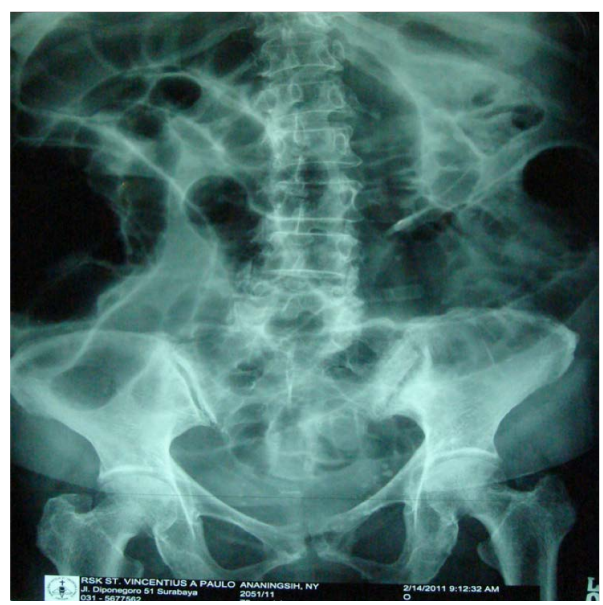

Figure 1. Plain abdominal X-Ray show dilatation of small and large intestine. 


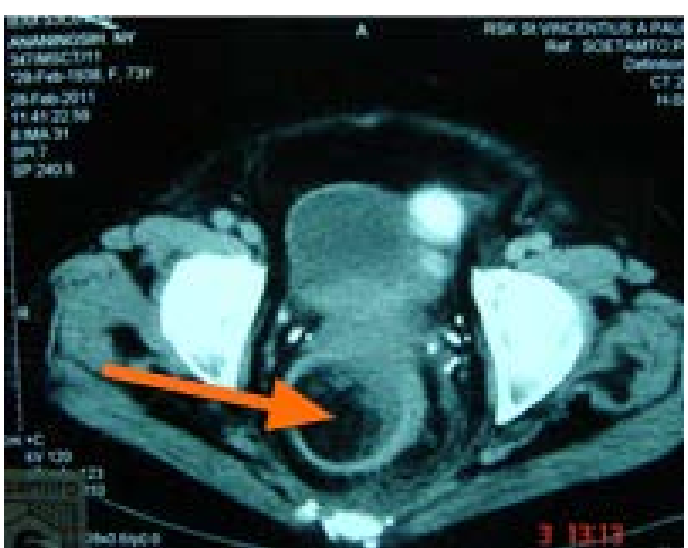

(a)

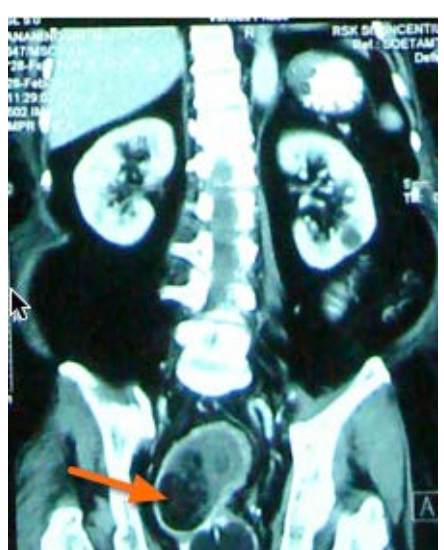

(b)

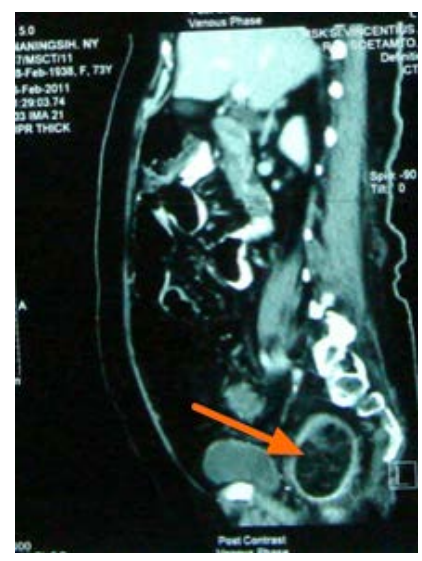

(c)

Figure 2. Axial, coronal, and sagittal CT image in the arterial phase show rectal tumor obstructed the rectum. (a) Axial view; (b) Coronal view; (c) Sagital view.

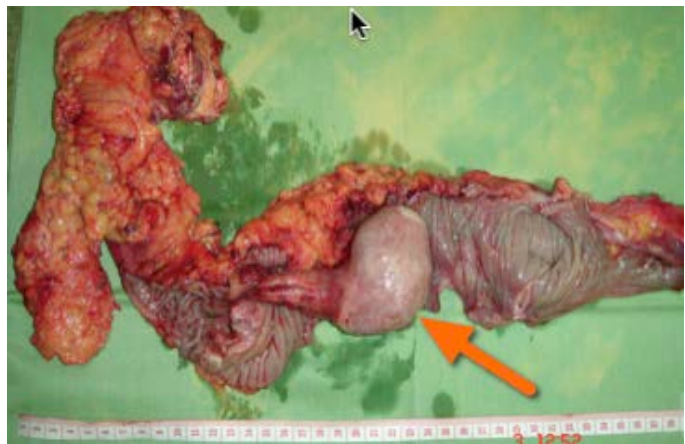

Figure 3. Intraluminalpolyp mass that invasive on the rectal and sigmoid wall.

\subsection{Case Report 2}

The case of S 54-year-old woman with chief complain is left lower abdominal pain and mass, Melena for one week, and history of repeating transfusion on hospital because of anemia. Physical examination mass diameter 9 $\mathrm{cm}$ on left lower abdomen, hard consistency and tenderness, fix. Endoscopic with normal limit.

MSCT are shown in Figure 4 find solid mass on left abdominal with $9 \mathrm{~cm}$ diameter, arterial feeding from artery lienalis Figure 5. FNAB: mesenchimal malignant tumor with differend diagnosis leomyosarcoma low grade and GIST. We decide to therapy with Glivec for 3 weeks. On follow up after Glivec therapy, physical examination find the mass consistency become soft and reduce the diameter become $7 \mathrm{~cm}$, mobile.

USG mass diameter reduce become $7.28 \times 3.59 \mathrm{~cm}$ (Figure 6), the problem is anemia (RBC $6.6 \mathrm{mg} / \mathrm{dl}$ ). We decided laparotomy exploration found tumor on descending colon extended to mesocolon, with peritoneal seeding $(++)$ (Figure 7), livernodule $(+)$ we performed left hemicolectomy. Pathologyis malignant mesenchymal tumor, mitosis 5/0, limfenode 6+/6 limfenode, nodular metastases (+) CD 117 positive. Patients received Glivec treatment for 1 year, in the second years patients died because of heart attack.

\subsection{Case Report 3}

A 20 year-old man was admitted to hospital because of urine retention and change bowel habit in 1 year, other complaints are frequent diarrhea since 1 year ago, sometimes defecation like goat droppings. Digital rectal sphincter tone obtained in the normal range, no enlarged prostate, no prostate nodules, palpable mass ekstralumen 1 - 6 o'clock direction, at a distance of $3 \mathrm{~cm}$ from the anal verge, the rectal mucosa slippery, and you do not get blood, mucus in handschoen. CEA within normal limits.

Examination of multislice CT scan are shown in Figure 8 looked solid extraluminal masses, round, demarcated in the presacral space measures $9.8 \times 10 \times 10 \mathrm{~cm}$, push down rektosigmoid and vescaurinaria to the anterior. 


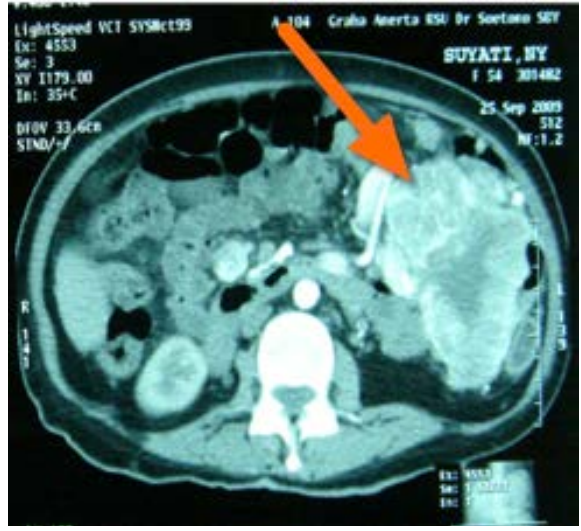

(a)

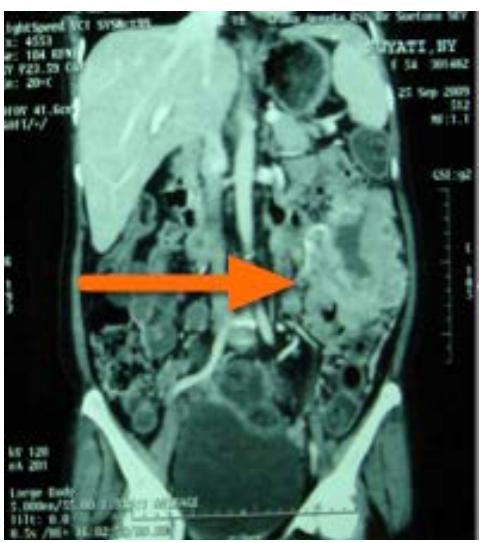

(b)

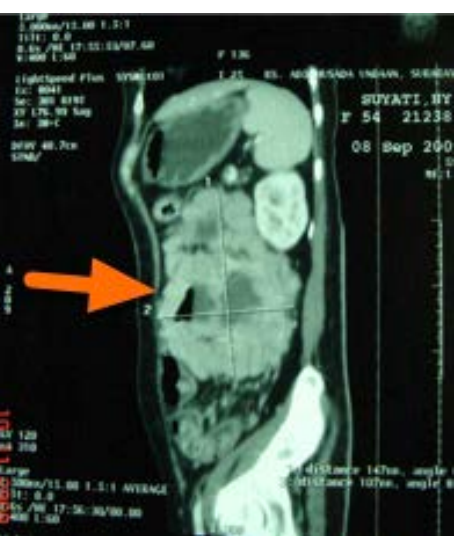

(c)

Figure 4. Axial, coronal, and sagittal CT image in the arterial phase show solid mass on left abdominal with $9 \mathrm{~cm}$ diameter. (a) Axial view; (b) Coronal view; (c) Sagittal view.

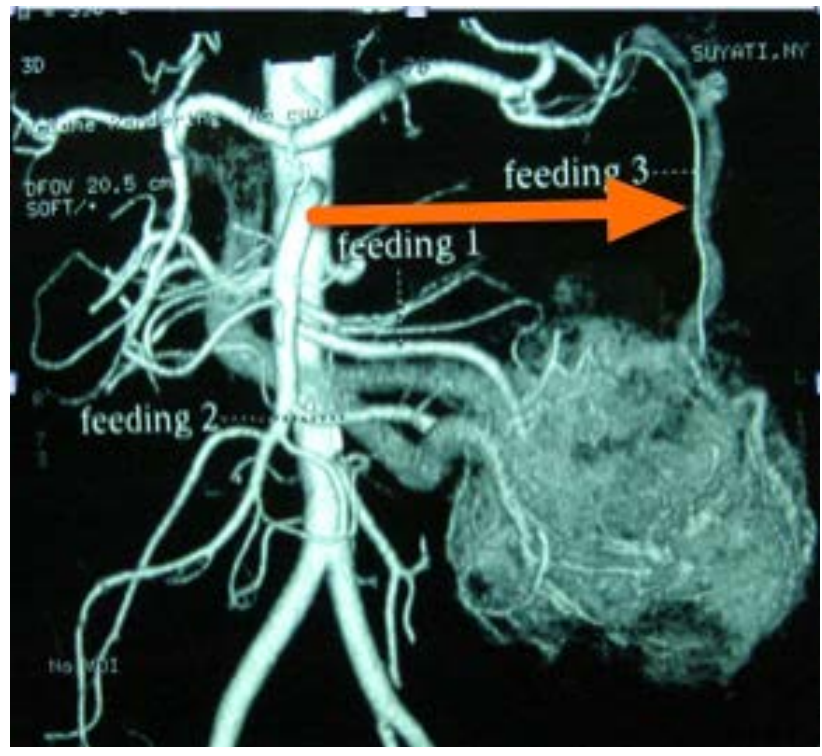

Figure 5. CT image in the arterial phase show solid mass on left abdominal with $9 \mathrm{~cm}$ diameter with feeding from artery lienalis.

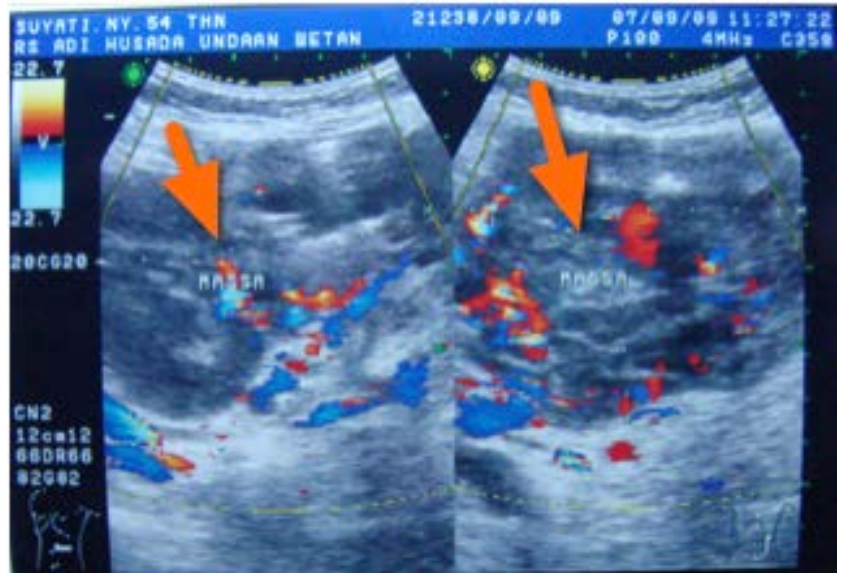

Figure 6. Abdominal ultra sonography with dopler shows the mass and vascular feeding reduce the diameter become $7 \mathrm{~cm}$. 


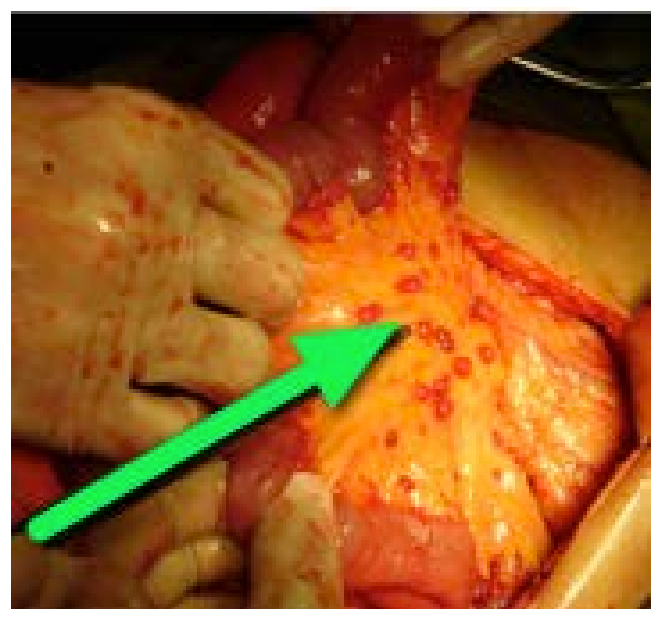

Figure 7. Intraoperative show descending colon mass with peritoneal seeding.

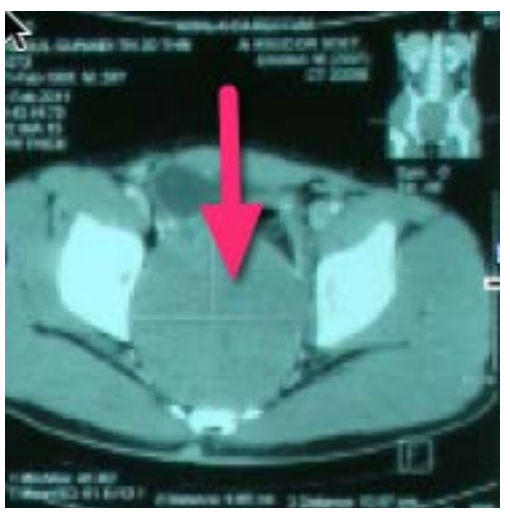

(a)

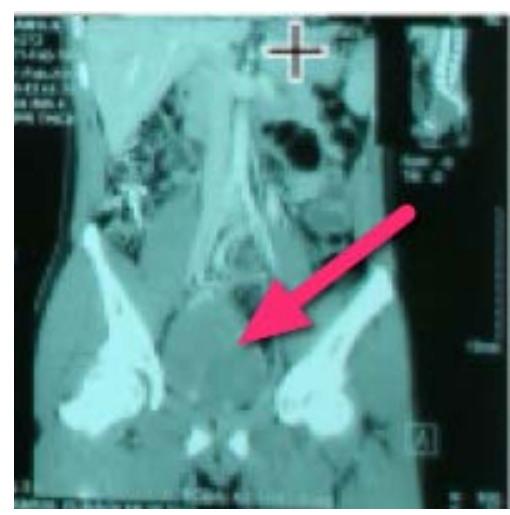

(b)

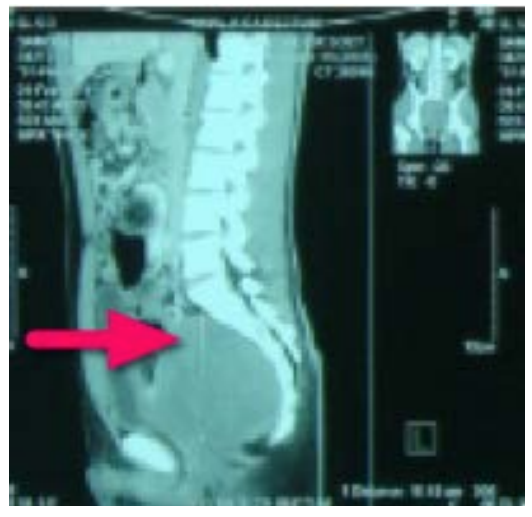

(c)

Figure 8. Axial, coronal, and sagittal CT image in the arterial phase show solid mass on pelvic region. (a) Axial view; (b) Coronal view; (c) Sagittal view.

There does not appear infiltration into the surrounding mass and enlarged lymph nodes and paraaorta and parailiaca. Examination guiding FNAB performed well with CT scan, the results of FNAB of the mass of pelvic cavity is spindle tumor mesechymal. colonoscopy with normal mucosa results. Caecum, colon ascendens, transverse colon, colon descenden, rectosigmoid not found mass, ulcer, erosions, polyps and diverticular. Patients also performed USG (Figure 9), colon in the loop and MRI examinations are shown Figure 10 found solid mass retrorektal. Patients diagnosed with suspicion retrorektal GIST (gastrointestinal stromal tumor).

Surgery performed with Milles procedure, found retrorektal tumor mass diameter of $10 \mathrm{~cm}$ that invasive on the rectal wall (Figure 11). The results of anatomic pathology is the gastro intestinal stromal tumor (GIST), high risk (tumor diameter $10 \mathrm{~cm}$, mitotic 6/50 hpf), infiltrative tumor growth in colon muscularispropria, found no metastasis at 9 limfenode were found and the results of immunohistochemical CD117 positive. Clinical evaluation of the first postoperative day obtained vital signs stable and within normal limits abdomen. Patient was scheduled for chemotherapy with Glivec. A month postoperative urinary incontinence complaints found, and that patients trained to CIC (intermittent catheterization) patient never buy the Glivec because of social problem and after that out of control.

\section{Discussion}

Colorectal GISTs are rare, frequency being reported at approximately 5\%, we just found three cases in 2 years, two localized at rectal and the other at descending Colon .Gist defined as cellular spindle cell, epithelioid, or pleomorphic mesenchymal tumor of the gastrointestinal (GI) tract, the term gastrointestinal stromal tumor 


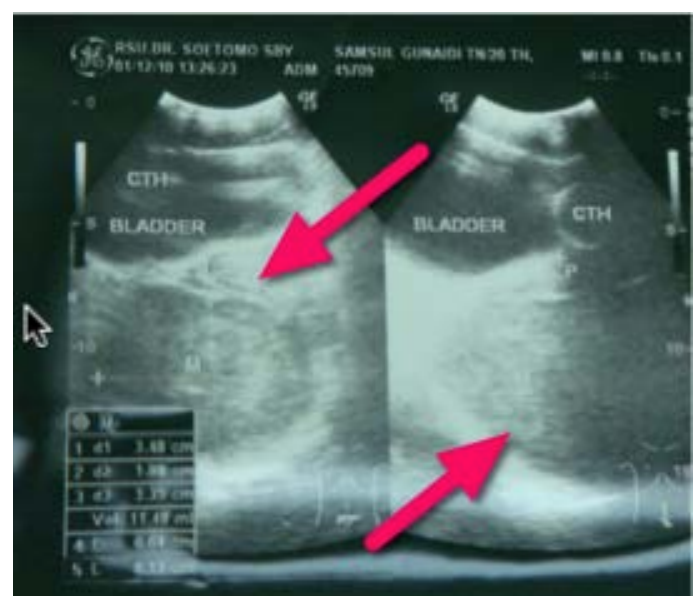

Figure 9. USG of the abdomen revealing a large solid mass pushing rektosigmoid and vesicaurinary.

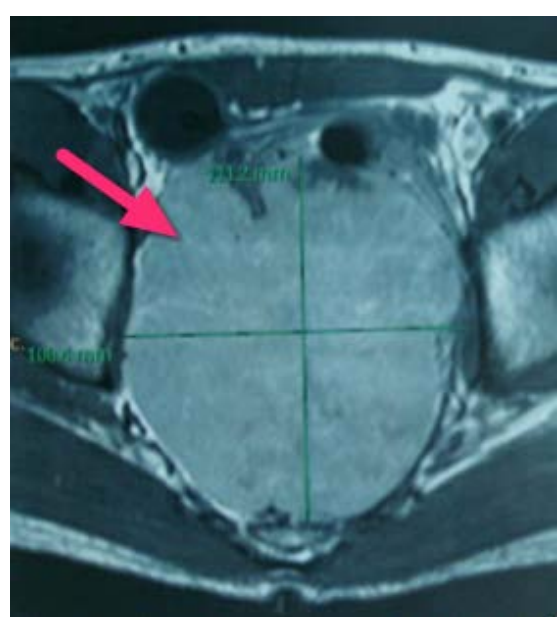

(a)

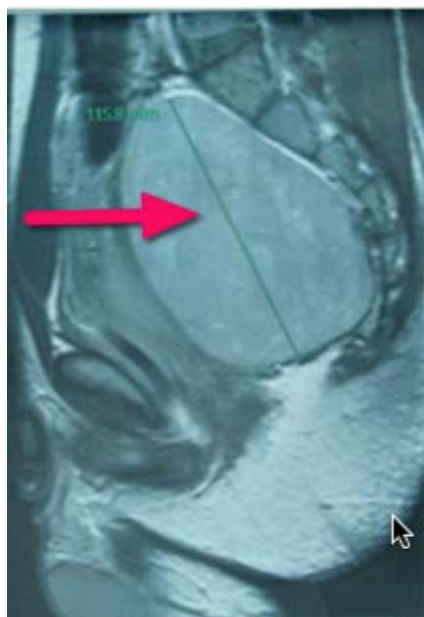

(b)

Figure 10. Axial and sagittal MRI image show solid retrorektamass. (a) Axial view 10; (b) Sagittal view.

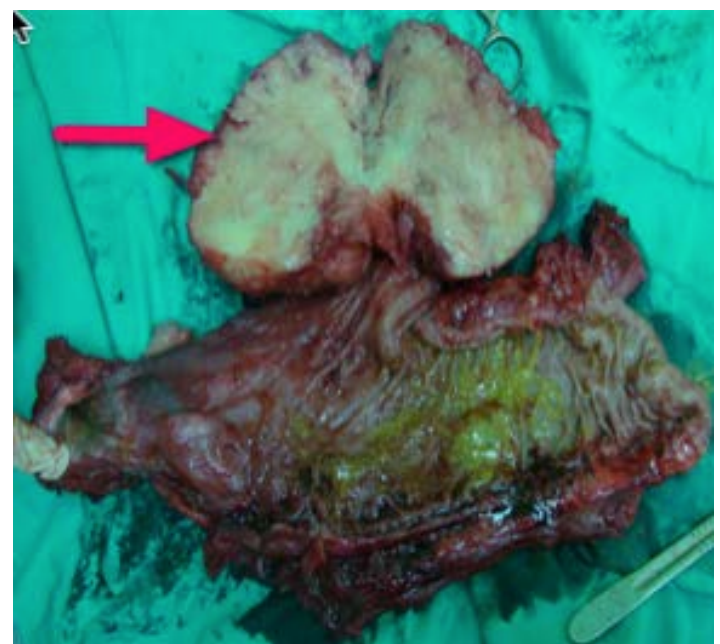

Figure 11. Rectorectal tumor mass that invasive on the rectal wall. 
(GIST) was introduced by Mazur and Clark in 1983 to differentiate GISTs from leiomyoma [1] [2]. The putative origin of these tumors is believed to be the interstitial cells of Cajal, the GI pacemaker cells and may arise anywhere in the gastrointestinal tract (GI). They are most common in the stomach (60\% - 70\%), followed by the small intestine $(20 \%$ - 25\%). Recently, they have also been described in extra-GI locations, principally mesentery, omentum, and retroperitoneum [1] [3].

According to all larger clinicopathologic series, GISTs have a predilection to adults over 50 years of age, with the median ages varying between 55 - 65 years in different sites with no clear sex predilection. Our study, 2 case over 50 years old and one case 20 years old, The proportion of patients under 40 years have ranged between 5\% and $20 \%$ in the larger clinicopathologic series [4].

Histological subtypes of GISTs include spindle, epithelioid or mixed cell tumors. In our surgery the histologi of three cases is myxoidlipo sarcoma, mesenchymal tumor, and gastro intestinal stromal tumor (GIST). The mass may be smooth or multinodular contour, containing central areas of hemorrhage, cystic change, necrosis or calcification. Even if the synchronous occurrence of GIST and Colorectal adenocarcinoma is not extremely rare, little is known about their potential common origin and carcinogenetic pathways [3] [7].

All cases we have presented had size more than $5 \mathrm{~cm}$ are considered as unfavorable prognostic factors to Fletcher criteria (Table 1): first case had High risk of aggressive behavior (size $8 \mathrm{~cm}$ and mitotic count $<5 / 50$ HPF), the second case had High risk of aggressive behavior (size $9 \mathrm{~cm}$ and mitotic count 5/10 HPF) and the third case had High risk of aggressive behavior (size $10 \mathrm{~cm}$ and mitotic count 6/50 HPF).

Surgical resection, for rectal gist performed low anterior resection and milles, descending colon GIST performed left hemicolectomy was performed for the only treatment. patients have no remained chief complain for several months after surgery and no recurrence has been observed, but one patient post milles with urinary incontinence complaints found and that patients trained to CIC (intermittent catheterization). The prognosis of GIST is aggressive and complete surgical for this reason, enbloc resection without tumor rupture is the treatment modality [2] [8].

GISTs are usually slow growing lesions, and are often incidentally discovered. Tumor size, location and growth pattern are associated with symptomatic presentation. Tumors smaller than $2 \mathrm{~cm}$ are often asymptomatic, usually limited to the colonic wall and appearing as submucosal masses in endoscopic studies. But in all our cases is advance size the smaller diameter is $8 \mathrm{~cm}$, obstruction and abdominal pain is the most common complication. Tung-Cheng write that the most common symptoms of rectal GIST are tenesmus and lower GI bleeding, which are similar to those of other kinds of rectal tumors [1] [8].

Immunohistochemically, GISTs are usually positive for KIT (CD117), a transmembrane receptor that is normally expressed on the myenteric plexus of the normal adult, among others tissues. All our cases immunocytochemical staining for CD117 is Positive. Approximately 70\% of GISTs coexpress CD34, and they also may be positive for smooth muscle actin and rarely for desmin and S-100 protein. However, the term GIST is only applied to gastrointestinal mesenchymal neoplasms witch are c-kit positive, with rare exceptions. Approximately 95\% of GISTs are positive for expression of the KIT (CD117, stem cell factor receptor) protein and as well as $70 \%-80 \%$ of GISTs expressing CD34, the human progenitor cell antigen [1]-[3]. Risk of malignancy is based on the primary tumor diameter and the mitotic count. A mitotic index over 5 per 50 high-power field (HPF)

Table 1. Proposed approach for assigning risk to gastrointestinal stromal tumors (Fletcher et al.).

\begin{tabular}{ccc}
\hline & \multicolumn{2}{c}{ Risk to Gastrointestinal Stromal Tumors } \\
\cline { 2 - 3 } & Size $(\mathrm{cm})$ & Mitotic Count (per 50 HPF $^{*}$ ) \\
\hline Very Low Risk & $<2$ & $>5$ \\
Low Risk & $2-5$ & $<-10$ \\
Intermediate Risk & $<5$ & $<5$ \\
Hight Risk & $5-10$ & $>5$ \\
& $>5$ & Any \\
& $>10$ & $>10$ \\
\hline
\end{tabular}

"HPF: Hight-Power Field. The Korean Journal of Pathology [10]. 
or size more than $5 \mathrm{~cm}$ are considered as unfavorable prognostic factors. The mitotic counts in GISTs vary in numbers from 0 - 20 per $50 \mathrm{HPF}$, and high mitotic counts are rare; the mitotic count is one of the most important histologic predictors of malignancy [4].

Anatomic location has been previously proposed as a prognostic factor independent of tumor size or mitotic rate. In this sense, small bowel tumors have been associated with the worst prognosis, while other authors have regarded most esophageal and colonic lesions as malignant. Unfortunately, tumor localization is not reliable in predicting outcome for each individual case. Because of some GISTs can recur or metastasize despite a histologically benign appearance, GISTs has been stratified into very low, low, intermediate and high-risk categories according to Fletcher criteria (tumor size and mitotic count). Actually, an indefinite follow up period has been suggested. Surgery is the standard treatment for localized primary disease without metastatic involvement, with a 5 -year survival range from $45 \%$ to $65 \%$. Endoscopic resection could be an option if technically feasible by tumor size or location but in our second case the rectal polyp size is the huge $(\phi 8 \mathrm{~cm})$ so we performed low anterior resection [1]-[3].

Liver and peritoneum are the most common metastatic sites in malignant GISTs. Lymph node metastases are very uncommon, in our second case have mesenterial node metastases. Glivec (Imatinib ${ }^{\circledR}$ ), a tyrosine kinase inhibitor, has been used with good results in metastatic or unresectable GISTs. Postoperative imatinib treatment is recommended if the tumor is removed grossly, but the operative specimen has positive microscopic margins, designated as R1 resection, or if a gross visible tumor was left behind designated as R2 resection. Observation is all that is recommended if an R0 resection (negative microscopic margins) was achieved. The consensus at this time is to treat patient in a multidisciplinary approach based on biopsy margin, tumor size, mitotic rate, site, immunohistochemical staining, and mutational status [9] in our second case the size reduce $2 \mathrm{~cm}$ on USG and CT examination before surgery. A phase III trial, ACOSOG Z9001, was the first to demonstrate that one year of imatinib as compared to placebo in the adjuvant setting, is effective in decreasing recurrences. The tumor size > $10 \mathrm{~cm}$ is a risk factor of prognosis in rectal GIST. The appropriate treatment for rectal GIST is complete resection without residual tumors, with complete removal of metastases even in patients with recurrence. Future studies should focus on neo-adjuvant treatment strategies [3] [8] [9].

\section{Conclusion}

Colorectal GIST is very rare and can present as mass abdomen. Complete resection with an aggressive attempt to remove all gross disease and achieve negative margins remains the fundamental surgical principle in the management of GIST. Most of GISTs are positive for expression of the CD117. Postoperative imatinib treatment is recommended.

\section{References}

[1] Macías-García, F., Parada, P., Martínez-Lesquereux, L., Pintos, E., Fraga, M. and Domínguez-Muñoz, J.E. (2010) Gastrointestinal Stromal Tumors (GISTs) of the Colon. Revista Espanola de Enfermedades Digestives, 102, 388-390. http://dx.doi.org/10.4321/S1130-01082010000600010

[2] Ibrahim, M., Mushtaq, C., Arshad, R. and Imtiyaz,, A.W. (2009) Extra Luminal Colonic Gastrointestinal Stromal Tumor : A Case Report. Cases Journal, 2, 7525. http://dx.doi.org/10.1186/1757-1626-2-7525

[3] Kinshuk, K., Corwyn, R., Calvin, L. and Yoo-Joung, K. (2011) Coexistence of Gastrointestinal Stromal Tumour and Colorectal Adenocarcinoma: Two Case Reports. Journal of Gastrointestinal Oncology, 2, 50-54.

[4] Markku, M. and Jerzy, L. (2003) Gastrointestinal Stromal Tumors (GISTs): Definition, Occurrence, Pathology, Differential Diagnosis and Molecular Genetics. Polish Journal of Pathology, 54, 3-24.

[5] Şerife, U. and Zafer, K. (2009) Radiologic Findings in Malignant Gastrointestinal Stromal Tumors. Diagnostic and Interventional Radiology, 15, 121-126.

[6] Gupta, S., Punia, R.S. and Kaushik, R. (2004) Gastrointestinal Stromal Tumour of the Colon Presenting with Intestinal Obstruction. Indian Journal of Cancer, 41.

[7] Venkat, K., Shashirekha, C.A., Suresh, T. and Jothinder, S. (2012) A Rare Case of Perforated Gist (Gastro Intestinal Stromal Tumours) of Transverse Mesocolon with Synchronus Rectal Carcinoma: A Case Report. International Journal of Biomedical Research, 3, 262-264.

[8] Tung-Cheng, C., Jin-Tung, L., Ben-Ren, L. and John, H. (2009) Oncological Results for the Surgical Treatment of Rectal Gastrointestinal Stromal Tumor. International Society of University Colon and Rectal Surgeons (Taiwan), 20, 
87-93.

[9] Christopher, B.T., Wanqing, Z., Ghulamullah, S. and Paul, M. (2012) Gastrointestinal Stromal Tumors: A Review of Case Reports, Diagnosis, Treatment, and Future Direction. ISRN Gastroenterology, 2012, Article ID: 595968.

[10] Jung, E.S., Kang, Y.K., Cho, M., Kim, J.M., Lee, W.A., Lee, H.E., Park, S.H., Sohn, J.H. and Jin, S.Y. (2012) Update on the Proposal for Creating a Guidelines for Cancer Registration of the Gastrointetinal Tumors (1-2). The Korean Journal of Pathology, 46, 443-453. 